\title{
Clinical Characteristics and Survival Outcomes of Infiltrating Lobular Carcinoma: A Retrospective Study of 365 Cases in China
}

\author{
Boyue $\operatorname{Han}^{1,2, *}$, Zhangyuan $\mathrm{Gu}^{3, *}$, Zhebin Liu ${ }^{1,2}$, Hong Ling $\mathbb{D}^{1,2}$ \\ 'Department of Breast Surgery, Fudan University Shanghai Cancer Center, Fudan University, Shanghai, 200032, People's Republic of China; \\ ${ }^{2}$ Department of Oncology, Shanghai Medical College, Fudan University, Shanghai, 200032, People's Republic of China; ${ }^{3}$ Department of Breast Surgery, \\ Shanghai First Maternity and Infant Hospital, Tongji University School of Medicine, Shanghai, 200040, People's Republic of China \\ *These authors contributed equally to this work
}

Correspondence: Zhebin Liu; Hong Ling, Email zhebinliu@shca.org.cn; linghong98@aliyun.com

\begin{abstract}
Purpose: The objective of this study was to compare the demographic characteristics, clinicopathological factors and survival outcomes between infiltrating lobular carcinoma (ILC) and infiltrating ductal carcinoma (IDC) using our single-center database.

Methods: Seventeen thousand two hundred and three breast cancer patients were treated at Fudan University Shanghai Cancer Center (FUSCC) from January 2000 to December 2017. We identified 365 cases with ILC and 16,838 cases with IDC. The Pearson chi-square test was used to compare tumor characteristics, and the Kaplan-Meier methods were used to perform the survival analysis.

Results: ILC had some distinctive characteristics from IDC such as older age (ranged from 61 to 80 : ILC $26.8 \%$ vs IDC $19.9 \%$, P $<0.001$; over 80: ILC $1.6 \%$ vs IDC $0.8 \%, \mathrm{P}<0.001$ ), larger tumor size (ranged from 2 to 5 : ILC $45.2 \%$ vs IDC $37.1 \%, \mathrm{P}=0.011$ ), much more hormone receptor expression (ILC 92.9\% vs IDC 73.0\%, P <0.001), extremely less HER-2 expression (ILC 7.1\% vs IDC 25.9\%, P < 0.001). The overall survival and disease-free survival of ILC were worse than IDC (5-year OS, ILC 93.6\% vs IDC 94.5\%, P < 0.001; 5-year DFS, ILC $88.5 \%$ vs IDC $91.6 \%, \mathrm{P}=0.008)$. It was worth noting that the ILC patients had a worse overall survival than IDC patients after our propensity score matching study $(\mathrm{P}=0.037)$. The univariate analysis concluded that positive HR (hormone receptor), high expression of Ki67 and higher pathologic tumor stage were poor prognostic markers of ILC. Multivariate analysis demonstrated that tumor stage was a poor prognostic marker after adjustment for the effects of the above three factors. The most common primary site of metastasis was bone, but the proportion in the ILC group was much higher than that in the IDC group $(56.25 \%$ vs $36.40 \%, \mathrm{P}=0.003)$.
\end{abstract}

Conclusion: Compared with IDC, ILC survived worse and was more prone to bone metastasis. Therefore, a comprehensive understanding of ILC and specific treatments are needed for further research.

Keywords: infiltrating lobular carcinoma, infiltrating ductal carcinoma, survival, prognosis

\section{Background}

Breast cancer is a heterogeneous disease composed of different pathological subtypes. ${ }^{1,2}$ Infiltrating lobular carcinoma (ILC) accounts for $10-15 \%$ of all breast cancers. ${ }^{3,4}$ Compared with the predominant histological subtype, infiltrating ductal carcinoma (IDC), ILC is recognized as a distinct and understudied disease. ${ }^{4,5}$ ILC tended to occur in older women. $^{6-8}$ Especially, the incidence of ILC was linked to hormone replacement therapy among postmenopausal patients. $^{9}$

Previous studies suggested that ILC was generally more challenging to visualize or palpate than IDC, clinically and mammographically. ${ }^{10,11}$ The prognosis of ILC has been described as either better or no different from IDCs, ${ }^{10,12,13}$ so a much deeper understanding of the clinical outcome is needed. ${ }^{14}$ 
To investigate clinical features and survival outcomes of ILC, we undertook a comparison of ILC and IDC using our single-center database. We aim to provide a more comprehensive and authentic assessment of the biological phenotypes and clinical characteristics, providing helpful information for clinical therapeutic strategies.

\section{Patients and Methods}

\section{Participant Eligibility and Data Collection}

The medical records of patients treated from January 2000 to December 2017 at the breast surgery department of FUSCC were reviewed. To analyze the clinical and pathological characteristics of ILC and IDC patients, we studied many variables, including the age of the patients, pathologic tumor size, lymph node status, histological grade, estrogen receptor (ER) and progesterone receptor (PR) status, expression of human epidermal growth factor receptor-2 (HER-2), expression of $\mathrm{Ki}-67$, the surgery type and other treatments (adjuvant/neoadjuvant chemotherapy, radiotherapy, endocrine therapy and target therapy). ER or PR positive were considered HR (hormone receptor) positive status. ${ }^{15}$ The proportion of metastasis sites (such as bone, brain, liver, lung, lymph nodes, ovary) and the site counts in ILC and IDC were calculated separately.

\section{Statistical Analysis}

Comparisons of clinical characteristics between those two groups were examined by using Pearson Chi-square tests. The primary endpoint for this study was overall survival (OS) and disease-free survival (DFS). OS was defined as the number of months from diagnosis to the date of death from any causes. Disease-free survival (DFS) was defined as the time between the first date of diagnosis to any locoregional recurrence, including ipsilateral breast, local/regional lymph nodes of the disease, contralateral breast cancer, any distant metastasis of the disease, or any secondary malignancy, whichever occurred first. OS and DFS curves were obtained using the Kaplan-Meier methods. The impacts of relative factors were assessed in univariate and multivariable Cox proportional hazards models, such as the age, cancer stage, hormone receptor status, of potential prognostic value in correlation with patient survival. A two-sided p-value less than 0.05 was considered statistically significant for all tests. Statistical analyses were performed using SPSS statistical software version 25.0 packages (IBM Corporation, Armonk, NY, USA). Propensity score matching (PSM) was employed (Match Ratio: 1:3) using $\mathrm{R}$ software version 3.5.3. (The $\mathrm{R}$ Project for Statistical Computing, https://www.r-project.org/). The R packages "MatchIt", "survminer", "cmprsk", and "foreign" with the appropriate libraries were used.

\section{Results}

\section{General Information}

The clinical characteristics of the two histological subtypes are summarized in Table 1. In this study, there were 17,203 female breast cancer patients enrolled. Three hundred sixty-five patients $(2.12 \%)$ in total patients were diagnosed as infiltrating lobular carcinoma of the breast (ILC group), and 16,838 patients $(97.88 \%)$ were diagnosed as infiltrating ductal carcinoma (IDC group).

The study showed a difference in age distribution between the ILC group and the IDC group. ILC patients were usually older than IDC patients, especially in the group whose age ranged from 61 to 80 (ILC 26.8\% vs IDC 19.9\%, $\mathrm{P}<0.001$ ) and in the group whose age was over 80 (ILC $1.6 \%$ vs IDC $0.8 \%, \mathrm{P}<0.001$ ).

\section{Tumor Characteristics}

Tumor size of ILC patients was usually larger than the tumor size of IDC patients, especially in the group whose tumor dimension ranged from 2 to 5 (ILC $45.2 \%$ vs IDC $37.1 \%, \mathrm{P}=0.011$ ). The ILC group tended to have more lymph nodes metastasis than the IDC group (N3: ILC $13.7 \%$ vs IDC $5.8 \%, \mathrm{P}<0.01$ ). The proportion of elevated Ki-67 group of ILC patients was much less than that of IDC patients (ILC $25.5 \%$ vs IDC $43.1 \%, \mathrm{P}<0.001$ ). Besides, patients with ILC had a higher HR rate (ILC 92.9\% vs IDC 73.0\%, P < 0.001) and lower HER-2 expression rate (ILC $7.1 \%$ vs IDC $25.9 \%, \mathrm{P}<0.001$ ). 
Table I Clinicopathological Characteristics of ILC Patients and IDC Patients

\begin{tabular}{|c|c|c|c|c|c|}
\hline \multirow{3}{*}{$\begin{array}{l}\text { Clinicopathological Characteristics and } \\
\text { Treatments }\end{array}$} & \multicolumn{2}{|c|}{ ILC Patients } & \multicolumn{2}{|c|}{ IDC Patients } & \multirow[b]{3}{*}{$p^{\mathbf{b}}$} \\
\hline & \multicolumn{2}{|c|}{$(n=365)$} & \multicolumn{2}{|c|}{$(n=16,838)$} & \\
\hline & No. & $\%$ & No. & $\%$ & \\
\hline Age (Year) & & & & & $<0.001$ \\
\hline $18-40$ & 31 & $8.5 \%$ & 2751 & $16.3 \%$ & \\
\hline $4 I-60$ & 230 & $63.0 \%$ & $10,60 \mid$ & $63.0 \%$ & \\
\hline $61-80$ & 98 & $26.8 \%$ & 3351 & $19.9 \%$ & \\
\hline$>80$ & 6 & $1.6 \%$ & 135 & $0.8 \%$ & \\
\hline Size $(\mathrm{cm})$ & & & & & 0.011 \\
\hline$\leq 2$ & 164 & $44.9 \%$ & 8984 & $53.4 \%$ & \\
\hline $2-5$ & 165 & $45.2 \%$ & 6240 & $37.1 \%$ & \\
\hline$>5$ & 9 & $2.5 \%$ & 383 & $2.3 \%$ & \\
\hline Unknown & 27 & $7.4 \%$ & $123 \mid$ & $7.3 \%$ & \\
\hline Pathological Lymph Node Status & & & & & $<0.001$ \\
\hline N0 (no metastatic lymph node) & 202 & $55.3 \%$ & 10,262 & $60.9 \%$ & \\
\hline NI (I-3 metastatic lymph nodes) & 76 & $20.8 \%$ & 3925 & $23.3 \%$ & \\
\hline N2 (4-9 metastatic lymph nodes) & 35 & $9.6 \%$ & 1570 & $9.3 \%$ & \\
\hline N3 (more than 10 metastatic lymph nodes) & 50 & $13.7 \%$ & 976 & $5.8 \%$ & \\
\hline Unknown & 2 & $0.5 \%$ & 105 & $0.6 \%$ & \\
\hline Hormone Receptor ${ }^{a}$ & & & & & $<0.001$ \\
\hline Positive & 339 & $92.9 \%$ & 12,290 & $73.0 \%$ & \\
\hline Negative & 26 & $7.1 \%$ & 4548 & $27.0 \%$ & \\
\hline HER-2 & & & & & $<0.001$ \\
\hline Positive & 26 & $7.1 \%$ & 4369 & $25.9 \%$ & \\
\hline Negative & 334 & $91.5 \%$ & $\mathrm{II}, \mathrm{I72}$ & $66.3 \%$ & \\
\hline Ki-67 & & & & & $<0.001$ \\
\hline Low $(<14 \%)$ & 231 & $63.3 \%$ & 7175 & $42.6 \%$ & \\
\hline High ( $\geq 14 \%$ ) & 93 & $25.5 \%$ & 7249 & $43.1 \%$ & \\
\hline Unknown & 41 & $11.2 \%$ & 2414 & $14.3 \%$ & \\
\hline Surgery & & & & & 0.049 \\
\hline Breast Concservative Surgery & 55 & $15.1 \%$ & 3132 & $18.6 \%$ & \\
\hline Mastectomy & 310 & $84.9 \%$ & 13,706 & $81.4 \%$ & \\
\hline Adjuvant Chemotherapy & & & & & $<0.001$ \\
\hline Yes & 233 & $63.8 \%$ & 11,960 & $71.0 \%$ & \\
\hline No & 111 & $30.4 \%$ & 3609 & $21.4 \%$ & \\
\hline Unknown & 21 & $5.8 \%$ & 1269 & $7.5 \%$ & \\
\hline Chemotherapy Regiment & & & & & 0.279 \\
\hline Anthracycline & 43 & $11.8 \%$ & 2792 & $16.6 \%$ & \\
\hline Taxanes & 58 & $15.9 \%$ & 2416 & $14.3 \%$ & \\
\hline Anthracycline+Taxanes & 121 & $33.2 \%$ & 6224 & $37.0 \%$ & \\
\hline Others & 6 & $1.6 \%$ & 322 & $1.9 \%$ & \\
\hline Unknown & 5 & $1.4 \%$ & 206 & $1.2 \%$ & \\
\hline Radiotherapy & & & & & 0.048 \\
\hline Yes & 150 & $41.1 \%$ & 6042 & $35.9 \%$ & \\
\hline No & 192 & $52.6 \%$ & 9276 & $55.1 \%$ & \\
\hline Endocrine Therapy & & & & & $<0.001$ \\
\hline Yes & 292 & $80.0 \%$ & 10,593 & $62.9 \%$ & \\
\hline No & 36 & $9.9 \%$ & 4767 & $28.3 \%$ & \\
\hline Unknown & 37 & $10.1 \%$ & 1478 & $8.8 \%$ & \\
\hline
\end{tabular}


Table I (Continued).

\begin{tabular}{|c|c|c|c|c|c|}
\hline \multirow{3}{*}{$\begin{array}{l}\text { Clinicopathological Characteristics and } \\
\text { Treatments }\end{array}$} & \multirow{2}{*}{\multicolumn{2}{|c|}{$\begin{array}{c}\text { ILC Patients } \\
(n=365)\end{array}$}} & \multirow{2}{*}{\multicolumn{2}{|c|}{$\begin{array}{c}\text { IDC Patients } \\
(n=\mid 6,838)\end{array}$}} & \multirow[b]{3}{*}{$p^{b}$} \\
\hline & & & & & \\
\hline & No. & $\%$ & No. & $\%$ & \\
\hline Target Therapy & & & & & $<0.001$ \\
\hline Yes & 18 & $4.9 \%$ & 2302 & $13.7 \%$ & \\
\hline No & 341 & $93.4 \%$ & 13,573 & $80.6 \%$ & \\
\hline Unknown & 6 & $1.6 \%$ & 963 & $5.7 \%$ & \\
\hline
\end{tabular}

Notes: ${ }^{a}$ HR positive: ER (estrogen receptor) positive or/and PR (progesterone receptor) positive. ${ }^{\mathrm{b}}$ Pearson Chi-square tests between IDC and ILC.

Abbreviations: HR, hormone receptor; HER-2, human epidermal growth factor receptor-2; ILC, infiltrating lobular carcinoma; IDC, infiltrating ductal carcinoma.

\section{Molecular Subtypes}

We exhibited a subtype distribution map for both groups in Figure 1. Compared with IDC, the ILC patients showed a discrepant distribution of molecular subgroups: luminal A breast cancer was the most frequent $(59 \%$ in ILC vs $30 \%$ in IDC, P $<0.001$ ), followed by luminal B breast cancer ( $23 \%$ in ILC vs $29 \%$ in IDC, P $<0.001$ ). The proportion of both triple-negative breast cancer (TNBC) and HER-2 overexpression breast cancer was much more prominent in the IDC patients $(6 \%$ in ILC vs $13 \%$ in IDC; $2 \%$ in ILC vs $12 \%$ in IDC, $\mathrm{P}<0.001)$ (Figure 1$)$.

\section{Treatments}

Compared with IDC group, fewer patients underwent breast conservative surgery (ILC $15.1 \%$ vs IDC $18.6 \%, \mathrm{P}=0.049$ ). This study also demonstrated that fewer percentages of ILC patients were handled with adjuvant chemotherapy (ILC $63.8 \%$ vs IDC $71.0 \%, \mathrm{P}<0.001$ ). Besides, more ILC patients received radiotherapy than IDC patients (ILC $41.1 \%$ vs IDC $35.9 \%, P<0.05)$.

According to the HR and HER-2 status between ILC and IDC patients, many more patients were treated with endocrine therapy in the ILC group (ILC $80.0 \%$ vs IDC $62.9 \%, \mathrm{P}<0.001$ ), while significantly fewer patients in the ILC group were treated with target therapy (ILC $4.9 \%$ vs IDC $13.7 \%, \mathrm{P}<0.001$ ).

A Molecular Subtype of ILC

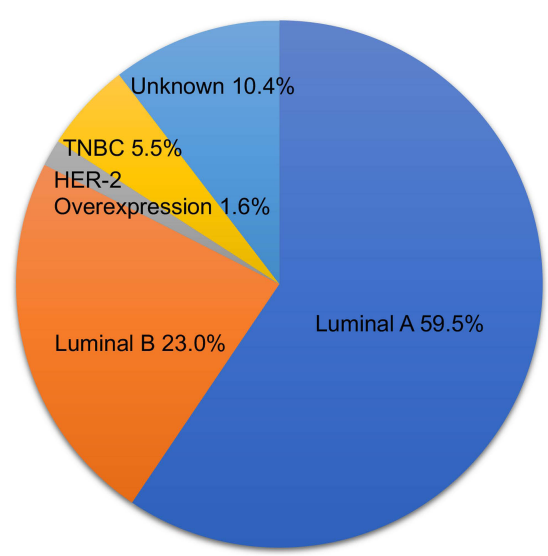

B

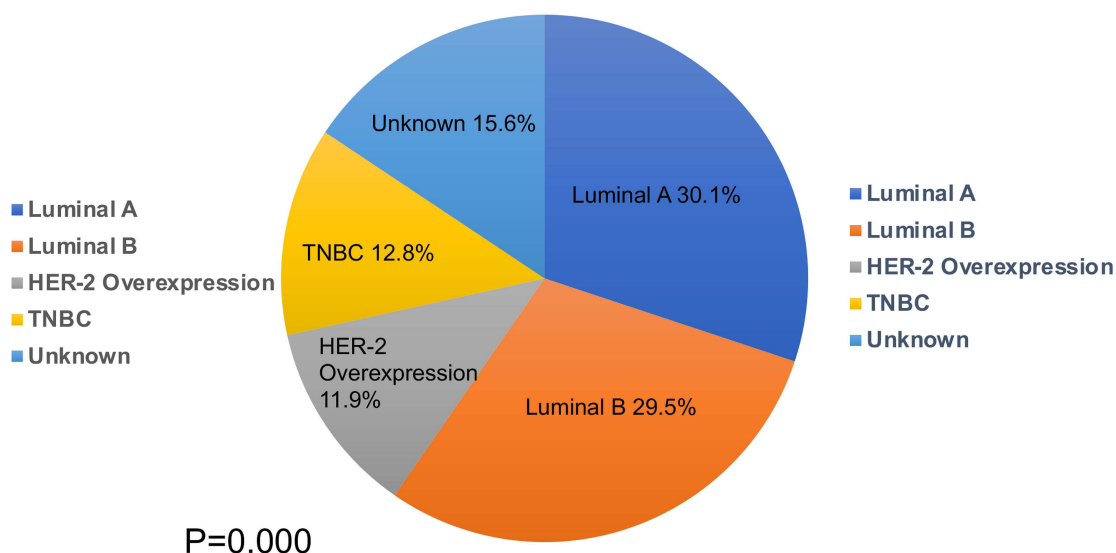

Figure I Molecular subtype distribution map for ILC and IDC group. (A) Molecular subtype of ILC group. (B) Molecular subtype of IDC group. (ILC vs IDC $P<0.00$ I, Log rank test). Luminal (A) ER+, PR+, HeR-2 (-), Ki-67 < 14\%; Luminal (B) HR+, Ki-67 14\%; HR+, HER-2 (+); ER+, PR-; Her-2 overexpression: HR (-), HER-2 (+); TNBC (Triple-negative breast cancer): ER (-), PR (-), HER-2 (-). 


\section{Survival Analysis}

In this study, the median follow-up duration was 65.7 months (mean, 68.7 months; range, 3 to 148 months). Figure 2 shows the OS and DFS curves. It showed that the ILC group had a worse survival than the IDC group. The OS in the ILC group was $93.6 \%$, while in the IDC group, it was $94.5 \%(\mathrm{P}<0.001)$. Regarding the DFS curve, the 5-year DFS of all ILC patients was $88.5 \%$, while in the IDC group was $91.6 \%(\mathrm{P}=0.008)$ (Figure 2). We further conducted DFS curves of the different molecular subgroups in the ILC and IDC cohorts. It was worth noting that IDC patients had a better prognosis than ILC patients in Luminal B $(\mathrm{P}=0.012)$ and TNBC subtype $(\mathrm{P}<0.001)$ (Figure S1).

Propensity score matching (PSM) was employed (Match Ratio: 1:3) to eliminate the bias of demographic and clinicopathological features between ILC and IDC groups (list of variables in Table 2). After matching, the 5-year OS of ILC was still worse than that of IDC $(\mathrm{P}=0.037)$ (Figure 3).

\section{Univariate and Multivariate Cox Regression Analysis in ILC Group}

In univariate Cox regression analysis, positive HR ( $\mathrm{P}<0.001)$, high expression of Ki-67 ( $\mathrm{P}=0.001)$, and cancer stage III $(\mathrm{P}<0.001)$ were correlated with significantly worse DFS in the ILC group. When these elements were put into a multivariate Cox regression analysis for ILC recurrence, cancer stage $(\mathrm{P}<0.001)$ and Ki-67 $(\mathrm{P}=0.017)$ were independent prognostic factors (Table 3).

It was remarkable that 5 -year OS showed a similar effect. In univariate Cox regression analysis, positive HR $(\mathrm{P}=$ 0.036), high expression of Ki-67 ( $\mathrm{P}=0.019)$, and cancer stage III $(\mathrm{P}<0.001)$ were associated with observably worse 5 -year OS in the ILC group. When these elements were put into a multivariate Cox regression analysis, cancer stage III was an independent prognostic factor $(\mathrm{P}<0.001)$ (Table 4).

\section{The Metastasis Sites in ILC Group and IDC Group}

Figure 4 shows the metastasis sites in the ILC group and IDC group. It demonstrated that the most common primary site of metastasis was bone, but the proportion in the ILC group was much higher than that in the IDC group (56.25\% vs $36.40 \%, \mathrm{P}=0.003)$. The liver was the second most common metastasis site $(12.50 \%)$, followed by the lymph nodes (8.33\%), lung (6.25\%) and brain (4.17\%) in the ILC group, while the order in the IDC group was the lung (25.30\%), liver $(15.48 \%)$, lymph nodes $(13.37 \%)$ and brain $(4.38 \%)(\mathrm{P}=0.003)$ (Table 5). We then compared the number of first metastasis sites in IDC and ILC, and found that there was no statistical difference (Table 6).

Table 7 shows the same second primary tumor occurrence trend after ILC and IDC. The top three malignant tumor happened were contralateral breast cancer ( $47.37 \%$ in ILC vs $37.39 \%$ in IDC), thyroid cancer $(21.05 \%$ in ILC vs $22.41 \%$ in IDC), and lung cancer (10.53\% in ILC vs $37.39 \%$ in IDC 14.87\%) $(\mathrm{P}=0.824)$.

A

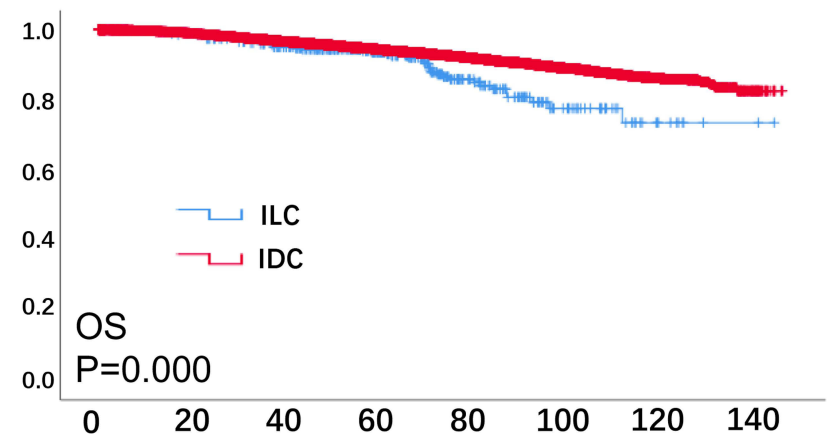

B

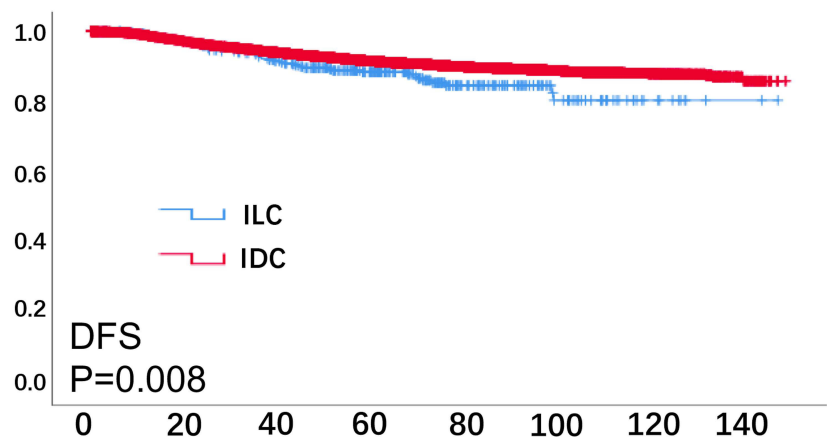

Figure 2 Kaplan-Meier curves illustrate OS and DFS for ILC and IDC in original samples. (A) OS Kaplan-Meier curves for ILC and IDC patients in original samples (ILC vs IDC P $<0.00$ I, Log rank test). (B) DFS Kaplan-Meier curve for ILC and IDC patients in original samples (ILC vs IDC P = 0.008, Log rank test). 
Table 2 Clinicopathological Characteristics of ILC Patients and IDC Patients After the Propensity Score Matching

\begin{tabular}{|c|c|c|c|c|c|}
\hline \multirow{3}{*}{$\begin{array}{l}\text { Clinicopathological Characteristics } \\
\text { and Treatments }\end{array}$} & \multicolumn{2}{|c|}{ ILC Patients } & \multicolumn{2}{|c|}{ IDC Patients } & \multirow[b]{3}{*}{$p^{\mathbf{b}}$} \\
\hline & \multicolumn{2}{|c|}{$(n=333)$} & \multicolumn{2}{|c|}{$(n=999)$} & \\
\hline & No. & $\%$ & No. & $\%$ & \\
\hline Age (year) & & & & & 0.924 \\
\hline $18-40$ & 22 & $6.6 \%$ & 75 & $7.5 \%$ & \\
\hline $4 I-60$ & 209 & $62.8 \%$ & 626 & $62.7 \%$ & \\
\hline $61-80$ & 97 & $29.1 \%$ & 286 & $28.6 \%$ & \\
\hline$>80$ & 5 & $1.5 \%$ & 12 & $1.2 \%$ & \\
\hline Size $(\mathrm{cm})$ & & & & & 0.727 \\
\hline$\leq 2$ & 162 & $48.6 \%$ & 474 & $47.4 \%$ & \\
\hline $2-5$ & 162 & $48.6 \%$ & 504 & $50.5 \%$ & \\
\hline$>5$ & 9 & $2.7 \%$ & 21 & $2.1 \%$ & \\
\hline Unknown & & & & & 0.463 \\
\hline Pathological Lymph Node Status & 185 & $55.6 \%$ & 556 & $55.7 \%$ & \\
\hline No (no metastatic lymph node) & 69 & $20.7 \%$ & 215 & $21.5 \%$ & \\
\hline NI (I-3 metastatic lymph nodes) & 34 & $10.2 \%$ & 121 & $12.1 \%$ & \\
\hline N2 (4-9 metastatic lymph nodes) & 45 & $13.5 \%$ & 107 & $10.7 \%$ & \\
\hline N3 (more than 10 metastatic lymph nodes) & & & & & 0.262 \\
\hline Unknown & 312 & $93.7 \%$ & 947 & $94.8 \%$ & \\
\hline Hormone Receptor ${ }^{a}$ & 21 & $6.3 \%$ & 52 & $5.2 \%$ & \\
\hline Positive & & $0.0 \%$ & & $0.0 \%$ & 0.46 \\
\hline Negative & 25 & $7.5 \%$ & 79 & $7.9 \%$ & \\
\hline HER-2 & 308 & $92.5 \%$ & 920 & $92.1 \%$ & \\
\hline Positive & & & & & 0.426 \\
\hline Negative & 247 & $74.2 \%$ & 748 & $74.9 \%$ & \\
\hline Ki-67 & 86 & $25.8 \%$ & 251 & $25.1 \%$ & \\
\hline Low $(<14 \%)$ & & & & & 0.924 \\
\hline High ( $\geq 14 \%)$ & 22 & $6.6 \%$ & 75 & $7.5 \%$ & \\
\hline Unknown & 209 & $62.8 \%$ & 626 & $62.7 \%$ & \\
\hline
\end{tabular}

Notes: a ${ }^{2}$ R positive: ER (estrogen receptor) positive or/and PR (progesterone receptor) positive. ${ }^{b}$ Pearson Chi-square tests between IDC and ILC.

Abbreviations: HR, hormone receptor; HER-2, human epidermal growth factor receptor-2; ILC, infiltrating lobular carcinoma; IDC, infiltrating ductal carcinoma.

\section{Discussion}

This study was a population-based study with over five years of follow-up time and a relatively large sample of ILC ( $\mathrm{n}=365)$ of a single-centre database. The study reflected that in Chinese women, the incidence of the age distribution of both ILC and IDC groups reached the peak in their 40's to 50's, and then it declined afterwards. Compared to patients with IDC, patients with ILC were older, especially in their 60 's to 70 's.

This study indicated that tumor size was more prominent in the ILC group, wildly when dimension $(\mathrm{cm})$ of tumor ranged from 2 to 5 . This conclusion stayed in step with the study of Lee et al. ${ }^{16}$ The absence of desmoplastic reaction might make the lesion of ILCs impalpable and invisible, as explained in the studies of Jung et al, Li et al and Arpino et al. ${ }^{9,17,18}$ That was also the reason why patients with ILC were diagnosed at a relatively late stage.

Our study revealed that ILC had a higher incidence of HR expression and a lower rate of HER-2 expression, ${ }^{19}$ which was consistent with the study of Soslow et al. Anti-HER-2 therapy is generally performed in HER-2 positive breast cancers ${ }^{20}$ however, the HER-2 overexpression rate was as low as $7.1 \%$. Clinical case reports have shown that patients with ERBB2-mutated breast cancers respond to targeted HER-2 therapy; ${ }^{21,22}$ and this was confirmed by responses to neratinib seen in ERBB2-mutated cancers in the SUMMIT trial. ${ }^{23}$ This emphasizes the importance of precision genetic sequencing, which can help provide therapy alterations. As clinical cancer sequencing becomes more routine, more ILC will benefit from targeted therapy. 


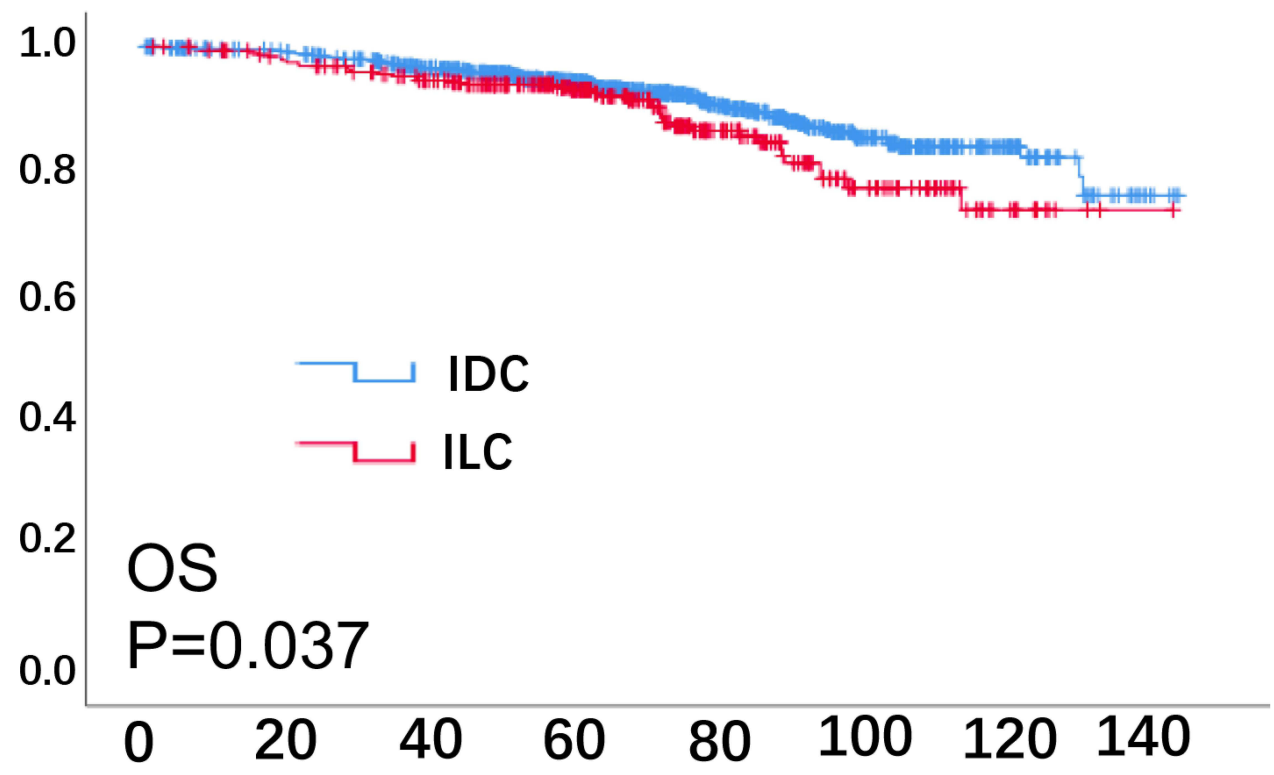

Figure 3 OS Kaplan-Meier curve for ILC and IDC after matching propensity score. (ILC vs IDC P = 0.037, Log rank test).

In agreement with the study of Pestalozzi et al, ${ }^{24}$ this study concluded that ILC was treated only a little less often with conservative breast surgery than IDC, probably because ILC was larger and referred to as a multicentric tumor. Previous studies reported that in terms of tumor downstaging, the benefits of neoadjuvant chemotherapy for ILC are limited. ${ }^{25,26}$ As the pCR (pathologic complete remission) rate for ILC is relatively low, ${ }^{27}$ locally advanced tumors, given the expected benefits, are minimal, consistent with the relatively low breast conservation rate.

As a general rule, the high expression of Ki-67 indicates that tumors are prone to recurrence and metastasis, which is a poor prognostic index, ${ }^{28,29}$ and has significant reference value for judging the prognosis of diseases. ${ }^{30-33}$ This study found that ILC had a lower Ki-67 score than IDC; thus, it explained the poor prognosis of ILC. The hallmark feature of

Table 3 Univariate Analysis and Multivariate Analysis for DFS in ILC Group

\begin{tabular}{|c|c|c|c|c|c|c|}
\hline \multirow[t]{2}{*}{ Variables } & \multicolumn{3}{|c|}{ Univariate } & \multicolumn{3}{|c|}{ Multivariate } \\
\hline & HR & $95 \% \mathrm{Cl}$ & $P$ value & HR & $95 \% \mathrm{Cl}$ & $P$ value \\
\hline Age (year) & & & 0.526 & & & \\
\hline$<40$ & I & & & & & \\
\hline$\geq 40$ & 0.223 & $0.187-1.48$ & & & & \\
\hline $\mathbf{H R}^{\mathrm{a}}$ & & & $<0.001$ & & & 0.066 \\
\hline Negative & I & & & I & & \\
\hline Positive & 0.225 & $0.104-0.487$ & & 0.448 & $0.190-1.056$ & \\
\hline Her-2 & & & 0.345 & & & \\
\hline Negative & I & & & & & \\
\hline Positive & 1.57 & $0.616-4.002$ & & & & \\
\hline Ki-67 & & & 0.001 & & & 0.017 \\
\hline Low & I & & & I & & \\
\hline High & 2.812 & $|.509-5.24|$ & & 2.242 & I.154-4.357 & \\
\hline Cancer Stage & & & $<0.001$ & & & 0.001 \\
\hline $0+1+I I$ & I & & & I & & \\
\hline III & 1.53 & I.247-I.877 & & 1.436 & $1.159-1.779$ & \\
\hline
\end{tabular}

Notes: ${ }^{a} \mathrm{HR}$ positive: ER (estrogen receptor) positive or/and PR (progesterone receptor) positive. Univariate analysis and multivariate analysis were performed by Cox regression model. The Univariate analysis included Age (year), HR, Her-2, Ki-67, cancer stage. The multivariate analysis included HR, Ki-67, Cancer Stage.

Abbreviations: HR, hazard ratio; $\mathrm{Cl}$, confidence interval; HR, hormone receptor; HER-2, human epidermal growth factor receptor-2; ILC, invasive lobular carcinoma; DFS, disease free survival. 
Table 4 Univariate Analysis and Multivariate Analysis for OS in ILC Group

\begin{tabular}{|c|c|c|c|c|c|c|}
\hline \multirow[t]{2}{*}{ Variables } & \multicolumn{3}{|c|}{ Univariate } & \multicolumn{3}{|c|}{ Multivariate } \\
\hline & HR & $95 \% \mathrm{Cl}$ & $P$ value & HR & $95 \% \mathrm{Cl}$ & $P$ value \\
\hline Age (year) & & & 0.409 & & & \\
\hline$<40$ & I & & & & & \\
\hline$\geq 40$ & 0.607 & $0.185-1.988$ & & & & \\
\hline $\mathbf{H R}^{\mathrm{a}}$ & & & 0.036 & & & 0.514 \\
\hline Negative & I & & & I & & \\
\hline Positive & 0.364 & $0.141-0.938$ & & 0.71 & $0.254-1.983$ & \\
\hline Her-2 & & & 0.500 & & & \\
\hline Negative & I & & & & & \\
\hline Positive & 0.612 & $0.147-2.545$ & & & & \\
\hline Ki-67 & & & 0.019 & & & 0.072 \\
\hline Low & I & & & I & & \\
\hline High & 2.276 & I. $148-4.515$ & & 1.945 & $0.943-4.011$ & \\
\hline Cancer Stage & & & $<0.001$ & & & $<0.001$ \\
\hline $0+1+11$ & I & & & I & & \\
\hline III & 1.577 & $1.275-1.95 \mid$ & & 1.533 & $1.233-1.908$ & \\
\hline
\end{tabular}

Notes: ${ }^{a} \mathrm{HR}$ positive: ER (estrogen receptor) positive or/and PR (progesterone receptor) positive. Univariate analysis and multivariate analysis were performed by Cox regression model. The Univariate analysis included Age (year), HR, Her-2, Ki-67, cancer stage. The multivariate analysis included HR, Ki-67, Cancer Stage.

Abbreviations: HR, hazard ratio; Cl, confidence interval; HR, hormone receptor; HER-2, human epidermal growth factor receptor-2; ILC, invasive lobular carcinoma; OS, overall survival.

cancer cells is uncontrolled division and reproduction, and many widely used clinical chemotherapeutics target this feature to prevent the rapid proliferation of cancer cells. It has been reported that mitotic activity, measured by the Ki-67 index, significantly reflected chemotherapy sensitivity. Besides, the advantage of chemotherapy is unclear for low Ki-67 patients. ${ }^{34}$ The study of Mathieu et al hinted that a lower Ki-67 score was a critical element associated with less adjuvant chemotherapy of ILC than IDC. ${ }^{35}$ Our results showed a high proportion of positive HR and low expression of Ki-67 in

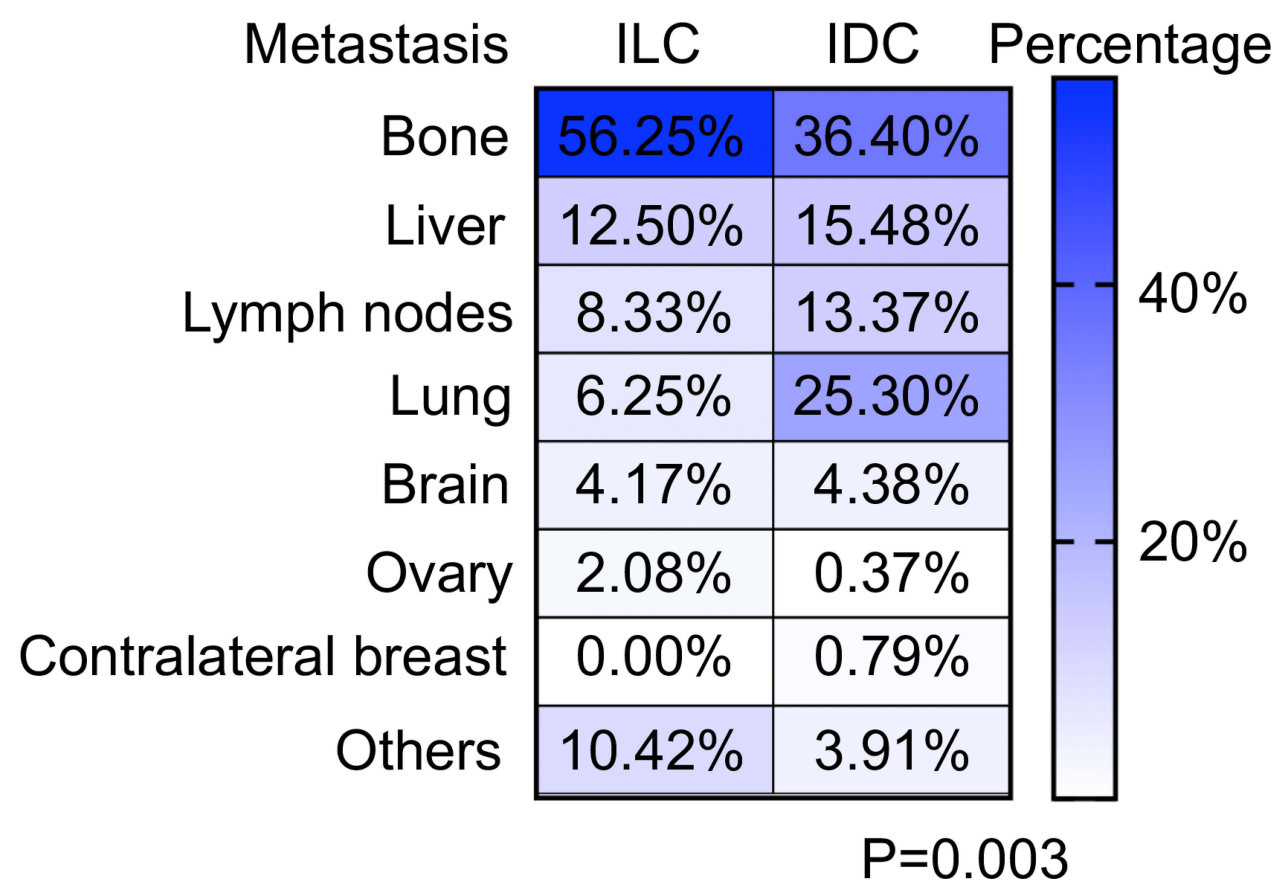

Figure 4 Metastasis sites of ILC patients and IDC patients. (ILC vs IDC P=0.003, Pearson Chi-square tests). Other metastatic sites include the kidney, Adrenal glands, Mesentery, Colorectal, eyeball, etc. The shades of blue represent the percentage of different metastasis sites. 
Table 5 Metastasis Sites of ILC Patients and IDC Patients

\begin{tabular}{|c|c|c|c|c|c|}
\hline \multirow{3}{*}{$\begin{array}{l}\text { Clinicopathological Characteristics and } \\
\text { Treatments }\end{array}$} & \multicolumn{2}{|c|}{ ILC Patients } & \multicolumn{2}{|c|}{ IDC Patients } & \multirow[b]{3}{*}{$p^{b}$} \\
\hline & \multicolumn{2}{|c|}{$(n=38)$} & \multicolumn{2}{|c|}{$(n=1309)$} & \\
\hline & No. & $\%$ & No. & $\%$ & \\
\hline Metastasis Sites & & & & & 0.003 \\
\hline Bone & 27 & $56.25 \%$ & 689 & $36.40 \%$ & \\
\hline Liver & 6 & $12.50 \%$ & 293 & $15.48 \%$ & \\
\hline Lymph nodes & 4 & $8.33 \%$ & 253 & $13.37 \%$ & \\
\hline Lung & 3 & $6.25 \%$ & 479 & $25.30 \%$ & \\
\hline Brain & 2 & $4.17 \%$ & 83 & $4.38 \%$ & \\
\hline Ovary & 1 & $2.08 \%$ & 7 & $0.37 \%$ & \\
\hline Contralateral breast & 0 & $0.00 \%$ & 15 & $0.79 \%$ & \\
\hline Others $^{\mathrm{a}}$ & 5 & $10.42 \%$ & 74 & $3.91 \%$ & \\
\hline
\end{tabular}

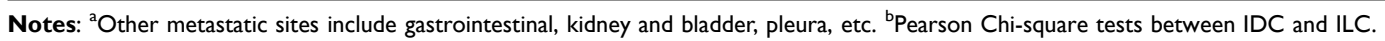
Abbreviations: ILC, infiltrating lobular carcinoma; IDC, infiltrating ductal carcinoma.

Table 6 Metastasis Site Counts of ILC Patients and IDC Patients

\begin{tabular}{|l|c|c|c|c|c|}
\hline \multirow{2}{*}{ Metastasis Site Counts $^{\mathbf{a}}$} & \multicolumn{2}{|c|}{ ILC Patients } & \multicolumn{2}{c|}{ IDC Patients } & \\
\cline { 2 - 6 } & No. & $\%$ & No. & $\%$ & $\boldsymbol{p}^{\mathbf{b}}$ \\
\hline & & & & & 0.332 \\
1 & 26 & $70.27 \%$ & 883 & $67.56 \%$ & \\
2 & 8 & $21.62 \%$ & 211 & $16.14 \%$ & \\
$\geq 3$ & 3 & $8.11 \%$ & 213 & $16.30 \%$ & \\
\hline
\end{tabular}

Notes: ${ }^{a}$ Metastasis site counts: the number of metastatic sites when metastasis first occurred. ${ }^{b}$ Pearson Chi-square tests between IDC and ILC. Abbreviations: ILC, infiltrating lobular carcinoma; IDC, infiltrating ductal carcinoma.

Table 7 Second Primary Tumor of ILC Patients and IDC Patients

\begin{tabular}{|l|c|c|c|c|c|}
\hline \multirow{2}{*}{ Second Primary Tumor } & \multicolumn{2}{|c|}{ ILC Patients } & \multicolumn{2}{c|}{ IDC Patients } & \multicolumn{1}{c|}{$\boldsymbol{p}^{\mathbf{c}}$} \\
\cline { 2 - 6 } & No. & $\%$ & No. & & 0.824 \\
Contralateral breast & & & & 377 & \\
Thyroid & 9 & $47.37 \%$ & 208 & $22.41 \%$ & \\
Lung & 4 & $21.05 \%$ & 138 & $14.87 \%$ & \\
Gynecology & 2 & $10.53 \%$ & 90 & $9.70 \%$ & \\
Gastrointestinal & 2 & $10.53 \%$ & 57 & $6.14 \%$ & \\
Kidney and bladder & 1 & $5.26 \%$ & 21 & $2.26 \%$ & \\
Others & 1 & $5.26 \%$ & 67 & $7.22 \%$ & \\
\hline
\end{tabular}

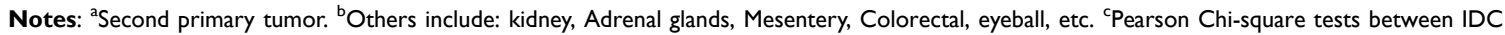
and ILC.

Abbreviations: ILC, infiltrating lobular carcinoma; IDC, infiltrating ductal carcinoma.

ILC, so it was supposed that most ILC patients were probably endocrine-sensitive but chemotherapy-insensitive. Therefore, it accounted for the lower proportion of chemotherapy and the large proportion of endocrine therapy in ILC patients.

In terms of prognosis, data showed that no matter 5-year OS or DFS, the prognosis of ILC patients was worse than that of IDC patients, the results are similar to those reported by Lee et $\mathrm{al}^{16}$ and Arpino et al. ${ }^{18}$ However, the study of Pestalozzi et al reported that the early-stage prognosis for ILC was better than that for IDC, while late-stage prognosis for 
ILC was worse. ${ }^{24}$ Previous studies showed the benefits of breast MRI for ILC, especially in the early detection of masses with irregular or spiculated margins or non-mass enhancing lesions. ${ }^{36}$ Inspired by this, we could try to use MRI to reduce the delayed diagnosis rate of ILC patients and improve the early-stage prognosis for ILC. Besides, the study of Jayasinghe et al reported that 10 -year survival of women with $84 \%$ for ILC, compared to IDC for $69 \%(\mathrm{p}=0.073){ }^{12}$ The difference of prognosis between this study and previous researches exposed a few limitations of this study. For instance, it was only a retrospective but not prospectively designed study. Besides, it was only a single-centre study. Nevertheless, in our study, when excluding prognosis factors by propensity score matching, it was worth noting that the prognosis of ILC was worse than that for IDC.

A previous study of Jayasinghe et al reported a higher risk ratio (RR) with young age at diagnosis, greater tumor size, higher pathologic stage and number of positive lymph nodes. ${ }^{12}$ In contrast, this study found that positive HR, high expression of Ki-67 and higher pathologic stage were independent prognostic indicators. After adjusting for the effects of the above three factors, survival declined with a higher pathologic stage.

As showed in Teo et al study, the sites of distant metastatic of ILC was different from that of IDC. ${ }^{37}$ In this study, ILC was less likely to affect livers and lungs than IDC did. Conversely, bone and brain were much more likely to be affected by ILC. An earlier study of Mathew et al found that after diagnosis of distant metastasis, there was no outcome difference between ILC and IDC, ${ }^{38}$ while the study of Blohmer et al showed that, after diagnosis of the first distant metastasis, survival was much shorter for ILC patients than IDC patients. ${ }^{39}$ The Cancer Genome Atlas (TCGA) research network recently published results of genomic characterization of 490 IDC and 127 ILC breast cancer cases. ${ }^{2,40}$ As expected, ILC was prone to luminal A subtype, CDH1 mutations, and loss of E-cadherin by mRNA expression. The E-cadherin gene functions prevent tumor invasion, ${ }^{41}$ and the loss of E-cadherin in ILC results in an increased capacity for tissue invasion. Therefore, the TCGA results provide possible molecular mechanisms for the different metastasis tendencies of ILC and IDC.

Because of the lack of relevant studies, ILC is treated in the same manner as IDC according to IDC's international group consensus. ${ }^{42}$ However, ILC is well established as a distinctive disease process, ${ }^{43}$ and our study demonstrated the distinct clinical characteristics and survival outcomes of ILC. So a "one size fits all" approach to therapy for all invasive breast carcinomas is not optimized for particular subtypes such as ILC. Thus, clinical trials designed to investigate improvements to the therapeutic management of ILC are needed.

\section{Conclusions}

In summary, ILC had some distinct characteristics from IDC, such as older age, larger tumor size, more positive HR expression and less HER-2 expression. Moreover, ILC survives worse than IDC. It demonstrated that bone metastasis in the ILC group was much higher than that in the IDC group. So we should pay attention to the treatment of bone metastases, which is an essential factor in controlling the development of ILC patients. For some advanced patients, genetic testing and targeted therapy is also the future direction we can work. In short, we expect that the comprehensive understanding of ILC will provide beneficial evidence for the clinicians and other clinical trials are necessary to conduct.

\section{Data Sharing Statement}

All data generated or analyzed during this study are reflected in the present published article [and its Supplementary Information].

\section{Ethics Statement}

This study did not involve animals. All procedures performed in studies involving human participants were following the ethical standards of the institutional and/or national research committee and with the 1964 Helsinki Declaration and its later amendments or comparable ethical standards. The Ethics Committee Review Board approved this retrospective study of Fudan University Shanghai Cancer Center (050432). The need to obtain informed consent was waived, as the study was a retrospective study, and there was no additional risk to patients. All data were anonymized to maintain patient privacy. 


\section{Author Contributions}

All authors made a significant contribution to the work reported, whether that is in the conception, study design, execution, acquisition of data, analysis and interpretation, or in all these areas; took part in drafting, revising or critically reviewing the article; gave final approval of the version to be published; have agreed on the journal to which the article has been submitted; and agree to be accountable for all aspects of the work.

\section{Funding}

There is no funding to report.

\section{Disclosure}

The authors state that they have no competing interests.

\section{References}

1. Sotiriou C, Pusztai L. Gene-expression signatures in breast cancer. $N$ Engl J Med. 2009;360(8):790-800. doi:10.1056/NEJMra0801289

2. Ciriello G, Gatza ML, Beck AH, et al. Comprehensive molecular portraits of invasive lobular breast cancer. Cell. 2015;163(2):506-519. doi:10.1016/j.cell.2015.09.033

3. McCart Reed AE, Kutasovic JR, Lakhani SR, Simpson PT. Invasive lobular carcinoma of the breast: morphology, biomarkers and 'omics. Breast Cancer Res. 2015;17:12. doi:10.1186/s13058-015-0519-x

4. Sikora MJ, Jankowitz RC, Dabbs DJ, Oesterreich S. Invasive lobular carcinoma of the breast: patient response to systemic endocrine therapy and hormone response in model systems. Steroids. 2013;78(6):568-575. doi:10.1016/j.steroids.2012.11.002

5. Tasdemir N, Ding K, Savariau L, et al. Proteomic and transcriptomic profiling identifies mediators of Anchorage-independent growth and roles of inhibitor of differentiation proteins in invasive lobular carcinoma. Sci Rep. 2020;10(1):11487. doi:10.1038/s41598-020-68141-9

6. Lyu H, Fu LM, Tu XY, et al. [Invasive breast lobular carcinoma with extracellular mucin: a clinicopathological analysis]. Zhonghua Bing Li Xue Za Zhi. 2019;48(10):779-783. Chinese. doi:10.3760/cma.j.issn.0529-5807.2019.10.006

7. de Kruijf EM, Bastiaannet E, Ruberta F, et al. Comparison of frequencies and prognostic effect of molecular subtypes between young and elderly breast cancer patients. Mol Oncol. 2014;8(5):1014-1025. doi:10.1016/j.molonc.2014.03.022

8. Li CI, Anderson BO, Porter P, Holt SK, Daling JR, Moe RE. Changing incidence rate of invasive lobular breast carcinoma among older women. Cancer. 2000;88(11):2561-2569. doi:10.1002/1097-0142(20000601)88:11<2561::AID-CNCR19>3.0.CO;2-X

9. Jung NY, Kim SH, Kim SH, et al. Effectiveness of breast MRI and (18) F-FDGPET/CT for the preoperative staging of invasive lobular carcinoma versus ductal carcinoma. J Breast Cancer. 2015;18(1):63-72. doi:10.4048/jbc.2015.18.1.63

10. Quan ML, Sclafani L, Heerdt AS, Fey JV, Morris EA, Borgen PI. Magnetic resonance imaging detects unsuspected disease in patients with invasive lobular cancer. Ann Surg Oncol. 2003;10(9):1048-1053. doi:10.1245/ASO.2003.03.016

11. Franceschini G, Manno A, Mule A, et al. Gastro-intestinal symptoms as clinical manifestation of peritoneal and retroperitoneal spread of an invasive lobular breast cancer: report of a case and review of the literature. BMC Cancer. 2006;6:193. doi:10.1186/1471-2407-6-193

12. Jayasinghe UW, Bilous AM, Boyages J. Is survival from infiltrating lobular carcinoma of the breast different from that of infiltrating ductal carcinoma? Breast J. 2007;13(5):479-485. doi:10.1111/j.1524-4741.2007.00468.x

13. Toikkanen S, Pylkkanen L, Joensuu H. Invasive lobular carcinoma of the breast has better short- and long-term survival than invasive ductal carcinoma. Br J Cancer. 1997;76(9):1234-1240. doi:10.1038/bjc.1997.540

14. Cao AY, Huang L, Wu J, et al. Tumour characteristics and the clinical outcome of invasive lobular carcinoma compared to infiltrating ductal carcinoma in a Chinese population. World J Surg Oncol. 2012;10:152. doi:10.1186/1477-7819-10-152

15. Hammond ME, Hayes DF, Wolff AC, Mangu PB, Temin S. American society of clinical oncology/college of American pathologists guideline recommendations for immunohistochemical testing of estrogen and progesterone receptors in breast cancer. J Oncol Pract. 2010;6(4):195-197. doi:10.1200/JOP.777003

16. Lee JH, Park S, Park HS, Park BW. Clinicopathological features of infiltrating lobular carcinomas comparing with infiltrating ductal carcinomas: a case control study. World J Surg Oncol. 2010;8:34. doi:10.1186/1477-7819-8-34

17. Li CI, Anderson BO, Daling JR, Moe RE. Trends in incidence rates of invasive lobular and ductal breast carcinoma. JAMA. 2003;289 (11):1421-1424. doi:10.1001/jama.289.11.1421

18. Arpino G, Bardou VJ, Clark GM, Elledge RM. Infiltrating lobular carcinoma of the breast: tumour characteristics and clinical outcome. Breast Cancer Res. 2004;6(3):R149-156. doi:10.1186/bcr767

19. Soslow RA, Carlson DL, Horenstein MG, Osborne MP. A comparison of cell cycle markers in well-differentiated lobular and ductal carcinomas. Breast Cancer Res Treat. 2000;61(2):161-170. doi:10.1023/A:1006479113769

20. Riedlinger GM, Joshi S, Hirshfield KM, Barnard N, Ganesan S. Targetable alterations in invasive pleomorphic lobular carcinoma of the breast. Breast Cancer Res. 2021;23(1):7. doi:10.1186/s13058-020-01385-5

21. Ali SM, Alpaugh RK, Downing SR, et al. Response of an ERBB2-mutated inflammatory breast carcinoma to human epidermal growth factor receptor 2-targeted therapy. J Clin Oncol. 2014;32(25):e88-91. doi:10.1200/JCO.2013.49.0599

22. Ben-Baruch NE, Bose R, Kavuri SM, Ma CX, Ellis MJ. HER2-mutated breast cancer responds to treatment with single-agent neratinib, a second-generation HER2/EGFR tyrosine kinase inhibitor. J Natl Compr Canc Netw. 2015;13(9):1061-1064. doi:10.6004/jnccn.2015.0131

23. Hyman DM, Piha-Paul SA, Won H, et al. HER kinase inhibition in patients with HER2- and HER3-mutant cancers. Nature. 2018;554 (7691):189-194. doi:10.1038/nature25475 
24. Pestalozzi BC, Zahrieh D, Mallon E, et al. Distinct clinical and prognostic features of infiltrating lobular carcinoma of the breast: combined results of 15 International Breast Cancer Study Group clinical trials. J Clin Oncol. 2008;26(18):3006-3014. doi:10.1200/JCO.2007.14.9336

25. Delpech Y, Coutant C, Hsu L, et al. Clinical benefit from neoadjuvant chemotherapy in oestrogen receptor-positive invasive ductal and lobular carcinomas. Br J Cancer. 2013;108(2):285-291. doi:10.1038/bjc.2012.557

26. Tsung K, Grobmyer SR, Tu C, Abraham J, Budd GT, Valente SA. Neoadjuvant systemic therapy in invasive lobular breast cancer: is it indicated? Am J Surg. 2018;215(3):509-512. doi:10.1016/j.amjsurg.2017.11.011

27. Thomas M, Kelly ED, Abraham J, Kruse M. Invasive lobular breast cancer: a review of pathogenesis, diagnosis, management, and future directions of early stage disease. Semin Oncol. 2019;46(2):121-132. doi:10.1053/j.seminoncol.2019.03.002

28. Robertson S, Stalhammar G, Darai-Ramqvist E, et al. Prognostic value of Ki67 analysed by cytology or histology in primary breast cancer. $J$ Clin Pathol. 2018;71(9):787-794. doi:10.1136/jclinpath-2017-204976

29. Ellis MJ, Suman VJ, Hoog J, et al. Ki67 proliferation index as a tool for chemotherapy decisions during and after neoadjuvant aromatase inhibitor treatment of breast cancer: results from the American College of Surgeons Oncology Group Z1031 trial (alliance). J Clin Oncol. 2017;35 (10):1061-1069. doi:10.1200/JCO.2016.69.4406

30. Van Asten K, Slembrouck L, Olbrecht S, et al. Prognostic value of the progesterone receptor by subtype in patients with estrogen receptor-positive, HER-2 negative breast cancer. Oncologist. 2019;24(2):165-171. doi:10.1634/theoncologist.2018-0176

31. Yuan P, Xu B, Wang C, Zhang C, Sun M, Yuan L. Ki-67 expression in luminal type breast cancer and its association with the clinicopathology of the cancer. Oncol Lett. 2016;11(3):2101-2105. doi:10.3892/ol.2016.4199

32. Besusparis J, Plancoulaine B, Rasmusson A, et al. Impact of tissue sampling on accuracy of Ki67 immunohistochemistry evaluation in breast cancer. Diagn Pathol. 2016;11(1):82. doi:10.1186/s13000-016-0525-z

33. Focke CM, Burger H, van Diest PJ, et al. Interlaboratory variability of Ki67 staining in breast cancer. Eur J Cancer. 2017;84:219-227. doi:10.1016/ j.ejca.2017.07.041

34. Zhu X, Chen L, Huang B, et al. The prognostic and predictive potential of Ki-67 in triple-negative breast cancer. Sci Rep. 2020;10(1):225. doi:10.1038/s41598-019-57094-3

35. Mathieu MC, Rouzier R, Llombart-Cussac A, et al. The poor responsiveness of infiltrating lobular breast carcinomas to neoadjuvant chemotherapy can be explained by their biological profile. Eur J Cancer. 2004;40(3):342-351. doi:10.1016/j.ejca.2003.08.015

36. Amin AL, Sack S, Larson KE, et al. Does the addition of breast MRI add value to the diagnostic workup of invasive lobular carcinoma? J Surg Res. 2021;257:144-152. doi:10.1016/j.jss.2020.07.035

37. Teo K, Gomez-Cuadrado L, Tenhagen M, et al. E-cadherin loss induces targetable autocrine activation of growth factor signalling in lobular breast cancer. Sci Rep. 2018;8(1):15454. doi:10.1038/s41598-018-33525-5

38. Mathew A, Rajagopal PS, Villgran V, et al. Distinct pattern of metastases in patients with invasive lobular carcinoma of the breast. Geburtshilfe Frauenheilkd. 2017;77(6):660-666. doi:10.1055/s-0043-109374

39. Blohmer M, Zhu L, Atkinson JM, et al. Patient treatment and outcome after breast cancer orbital and periorbital metastases: a comprehensive case series including analysis of lobular versus ductal tumour histology. Breast Cancer Res. 2020;22(1):70. doi:10.1186/s13058-020-01309-3

40. Metzger-Filho O, Ferreira AR, Jeselsohn R, et al. Mixed invasive ductal and lobular carcinoma of the breast: prognosis and the importance of histologic grade. Oncologist. 2019;24(7):e441-e449. doi:10.1634/theoncologist.2018-0363

41. Vleminckx K, Vakaet L, Mareel M, Fiers W, van Roy F. Genetic manipulation of E-cadherin expression by epithelial tumour cells reveals an invasion suppressor role. Cell. 1991;66(1):107-119. doi:10.1016/0092-8674(91)90143-M

42. Carcoforo P, Raiji MT, Langan RC, et al. Infiltrating lobular carcinoma of the breast presenting as gastrointestinal obstruction: a mini review. $J$ Cancer. 2012;3:328-332. doi:10.7150/jca.4735

43. McCart Reed AE, Kalinowski L, Simpson PT, Lakhani SR. Invasive lobular carcinoma of the breast: the increasing importance of this special subtype. Breast Cancer Res. 2021;23(1):6. doi:10.1186/s13058-020-01384-6

\section{Publish your work in this journal}

Cancer Management and Research is an international, peer-reviewed open access journal focusing on cancer research and the optimal use of preventative and integrated treatment interventions to achieve improved outcomes, enhanced survival and quality of life for the cancer patient. The manuscript management system is completely online and includes a very quick and fair peer-review system, which is all easy to use. Visit http://www.dovepress.com/testimonials.php to read real quotes from published authors.

Submit your manuscript here: https://www.dovepress.com/cancer-management-and-research-journal 